\title{
Clinical Evaluation of an Oral Electrolyte Solution Formulated Based on Strong Ion Difference (SID) and Using Propionate as the Organic Anion in the Treatment of Neonatal Diarrheic Calves with Strong Ion Acidosis*
}

\author{
Henry Stämpfli ${ }^{1}$, Olimpo Oliver ${ }^{2}$, John K. Pringle ${ }^{3}$ \\ ${ }^{1}$ Department of Clinical Studies, Ontario Veterinary College, University of Guelph, Guelph, Canada \\ ${ }^{2}$ Facultad de MedicinaVeterinaria y de Zootecnia, Universidad Nacional de Colombia, Bogota, Colombia \\ ${ }^{3}$ Department of Clinical Sciences, Swedish University of Agricultural Sciences, Uppsala, Sweden \\ Email: hstaempf@uoguelph.ca
}

Received November 14, 2011; revised December 4, 2011; accepted December 28, 2011

\begin{abstract}
Background: It is postulated that the concentrations of the major strong ions $(\mathrm{Na}, \mathrm{K}$, and $\mathrm{Cl})$ in oral electrolyte solutions play a major role in clinical efficacy of these solutions for rehydration and corrections of metabolic acid base derangements. Objectives: The purpose of this study was to test prospectively the efficacy of an OES (OES $\mathrm{exp})$ formulated based on concentration of strong ion difference (SID) and propionate in a group of calves with naturally occurring neonatal diarrhea and clinically detectable dehydration and acid base abnormalities. Animals: Ten client owned calves of varying breeds, 2 - 22 days old, presented to a veterinary teaching hospital with a history of naturally occurring acute undifferentiated diarrhea, progressive depression and dehydration for treatment. Methods: Clinical and laboratory parameters were measured pre and post two oral electrolyte treatments to assess efficacy of the experimental OES to correct clinical and clinico pathological parameters. For the clinical trial the calves served as their own controls. For control of safety of medication 4 normal calves were force fed $4 \mathrm{~L}$ of $\mathrm{OES}_{\exp }$ and followed over a 24 hour period. Results: All calves had severe diarrhea and metabolic acidosis. The metabolic acidosis observed in the plasma of these calves and reflected by $\mathrm{pH}, \mathrm{HCO}_{3}^{-}$, SID and base deficit was corrected significantly towards reference ranges $(\mathrm{p}<0.05)$ with two $2 \mathrm{~L}$ feedings 12 hours apart. Dehydration was significantly corrected and all calves were discharged 1 - 3 days post admission. Conclusion and Clinical Importance: The use of SID is a valid approach when formulating oral electrolytes solutions for use in calves with acute diarrhea and metabolic derangement. Sodium propionate is valid substitute for commonly used sodium base equivalents in North America in oral electrolyte solutions.
\end{abstract}

Keywords: Propionate; Acid-Base; Rehydration; Strong Ions

\section{Introduction}

Young calves with diarrhea are routinely treated with oral electrolyte solutions (OES) of different compositions $[1,2]$. These OES's are theoretically formulated to maximize fluid and electrolyte absorption, to correct fluid deficits and acid-base derangements, as well as to provide nutritional support [1-3]. The efficacy of different OES can be predicted from their composition $[1,4]$. The diarrhea results in net losses of water, sodium, potassium, chloride and bicarbonate inducing progressive dehydration, hypovolemia, electrolyte imbalance and strong ion

* Research Support: NSERC Canada (National Science and Research Council.

Conflict of Interest Statement: None of the authors of this paper has a financial or personal relationship with other people or organisations that could inappropriately influence or bias the content of the paper. acidosis [1-3]. Additionally, increased production of 1-lactate from tissues or d-lactate originating from intestinal microbes can contribute to the severity of strong ion acidosis [4]. Use of OES is indicated for the prevention or correction of dehydration and electrolyte imbalance [1-3]. Optimal rehydration depends on sodium absorption, as water molecules follow solute to expand the extra cellular fluid space (ECF) $[1,3]$. Traditionally, the alkalinizing ability of oral electrolytes to correct concurrent metabolic acidosis has been attributed to anions, mainly bicarbonate or its metabolizable precursors, such as acetate, 1-lactate, gluconate and citrate [1,2]. Alternatively, physicochemical or quantitative approach to acid-base interpretation of body fluid homeostasis incorporates laws of chemistry of watery solutions with the concept of strong independent completely dissociated electrolytes 
(mainly $\mathrm{Na}, \mathrm{Cl}, \mathrm{K}$, and Lactate), and weak dependent electrolytes (mainly hydronium ions and bicarbonate ions) [5]. The strong ion difference is defined for the ECF as difference between $\sum$ strong cations- $\sum$ strong anions and is used clinically in quantitative acid-base interpretation as $\mathrm{SID}=\left(\mathrm{Na}^{+}+\mathrm{K}^{+}\right)-\left(\mathrm{Cl}^{+}, \mathrm{Lac}^{-}\right)$. The role of strong electrolytes and proteins in acid base homeostasis has been neglected, as pathophysiologic interpretations of acid base disturbances were mainly based on the Henderson-Hasselbalch equation [3]. OES are watery solutions containing mainly the strong ions $\mathrm{Na}, \mathrm{K}$ and $\mathrm{Cl}$ in significant concentrations [6]. It has been shown that $\mathrm{pH}$ in watery solutions such as OES is determined by SID [6]. Therefore it can be mathematically shown that the alkalinizing ability of OES is mainly determined by the concentrations of the strong ions (sodium, potassium and chloride) [4,6]. Examination of the composition of commercially available oral electrolyte solutions and applying physicochemical principles to it reveals that the concentrations of the strong electrolytes represented as SID are major factors determining the in vivo alkalinity or acidity of an oral electrolyte solution [7]. From results of earlier studies comparing efficacy of 6 different OES in experimental calf diarrhea models it was evident that the higher the SID in the OES, the lower was the mortality $[4,8]$. Applying quantitative acid-base interpretation to OES would emphasize SID as an additional criterion for OES quality for treatment of calf diarrhea. Therefore it appears logical to use the strong ion concept to optimally formulate OES for treatment of dehydration and acidbase imbalances in calves with neonatal diarrhea and dehydration. It is proposed to shift the focus on strong cations, especially sodium and potassium, with the intention to achieve a higher SID. The goal for formulation of this OES was to have an osmolality of approximately 350 $\mathrm{mmol} / \mathrm{L}$, a high SID, at about $110 \mathrm{mmol} / \mathrm{L}$, and propionate at $110 \mathrm{mmol} / \mathrm{L}$, respectively. Propionate was the weak anion chosen because it is a source of energy, appears to enhance sodium absorption in the small intestine, does not interfere with abomasal $\mathrm{pH}$, and may even inhibit overgrowth of Salmonella species [1]. The purpose of this study was to test prospectively the efficacy of such an OES in a group of calves with naturally occurring undifferentiated neonatal diarrhea, clinically detectable dehydration and as serving as their own controls.

\section{Material and Methods}

Ten calves of varying breeds, 2 - 22 days old, presented to a veterinary teaching hospital with a history of naturally occurring acute undifferentiated diarrhea, progresssive depression and dehydration were included in the study. Only calves that were still able to stand or at least maintain sternal recumbency, had an empiric clinical dehydration of $\leq 8 \%$ and a detectable suckle reflex were admitted to the study. The calves served as their own controls. The clinical assessment at admission included a complete physical examination, rectal temperature, pulse, and respiration rates (Table 1). Clinical dehydration in \% of body weight using extent of enophthalmos, duration of skin tent of ocular palpebra and hydration of oral mucous membranes were recorded [8]. Depression, based on signs of weakness, reduction in suckle reflex or recumbency was scored as $1=$ mild; $2=$ moderate; and $3=$ severe. Duration of onset of diarrhea to presentation was recorded in hours. Severity of diarrhea was empirically assessed with $1=$ mild; $2=$ moderate; $3=$ severe. A pre-treatment venous blood sample, collected in sodium Lithium tubes, was obtained from the jugular vein and analysed using a NOVA-Statprofile 9 (Nova Biomedical Canada LTD) for blood gases and blood-electrolytes including blood lactate $\left(\mathrm{Na}^{+}, \mathrm{K}^{+}, \mathrm{Cl}^{-}, \mathrm{L}-\mathrm{Lac}^{-}\right)$. One aliquot was analysed for hematocrit with microcentrifugation and total protein concentrations using refractometry. Adequacy of passive transfer was assessed in all calves by the zinc-sulphate turbidity test [9]. One calf was treated for failure of passive transfer with $1 \mathrm{~L}$ of frozen plasma within the first 24 hours of admission and was retained in the study. Immediately following blood sampling each calf was tube-fed 2 L of the experimental OES $\left(\mathrm{OES}_{\exp }\right.$ ) (MTC Animal Health Cambridge Ontario), and this was repeated 12 hours later. No other oral or parenteral fluids were given to the calves during the study period. Each $\mathrm{OES}_{\text {exp }}$ used was prepared as per manufacturer suggestions using $2 \mathrm{~L}$ of distilled water and was analyzed for concentrations of $\mathrm{Na}^{+}, \mathrm{Cl}^{-}, \mathrm{K}^{+}$and for $\mathrm{pH}$ on the blood gas machine. The $\mathrm{OES}_{\text {exp }}$ used was proposed to have an osmolality of approximately 350 $\mathrm{mmol} / \mathrm{L}$, an SID at about $110 \mathrm{mmol} / \mathrm{L}$, with the cations

Table 1. Descriptive statistics of clinical variables of calves with diarrhea at admission.

\begin{tabular}{cc}
\hline Parameter & Mean $\pm \mathrm{SD}(\mathrm{n}=10)$ \\
\hline Age (Days) & $\mathbf{8 . 6} \pm \mathbf{4 . 5}$ \\
Dehydration $^{\mathrm{a}}(\% \mathrm{BW})$ & $\mathbf{6 . 5} \pm \mathbf{1 . 2}$ \\
Diarrhea (hours) & $\mathbf{4 6 . 8} \pm \mathbf{4 1 . 7}$ \\
Diarrhea score & $\mathbf{3 . 0} \pm \mathbf{0 . 0}$ \\
Depression score & $\mathbf{2 . 2} \pm \mathbf{0 . 4 2}$ \\
Heart rate $\left(\mathrm{b} / \mathrm{min}^{\mathrm{b}}\right)$ & $\mathbf{1 3 1} \pm \mathbf{2 1 . 2}$ \\
Resp rate $(\mathrm{B} / \mathrm{min})$ & $\mathbf{4 1 . 6} \pm \mathbf{1 6 . 2}$ \\
Temperature $\left({ }^{\circ} \mathrm{C}\right)$ & $\mathbf{3 9 . 1} \pm \mathbf{0 . 9}$ \\
\hline
\end{tabular}

a Assessed by estimating \% of dehydration using degree of enophthalmus, suprapalpebral skin tent and dryness of oral mucus membranes ${ }^{8}$. ${ }^{b}$ Scored at $1=$ mild, 2 = moderate, $3=$ severe. 
sodium and potassium at $140 \mathrm{mmol} / \mathrm{L}$ and $30 \mathrm{mmol} / \mathrm{L}$, and the anions chloride and propionate at $60 \mathrm{mmol} / \mathrm{L}$ and $110 \mathrm{mmol} / \mathrm{L}$, respectively. After 24 hours and before the third OES $_{\text {exp }}$ feeding, a second venous blood sample was obtained for reassessment of acid-base values, electrolytes, microhematocrit and total protein concentrations. The calves were monitored regularly and observations recorded on an intensive care unit (ICU) form. Statistix 4.1 was used to analyse the data and to develop descriptive statistics. Values of laboratory variables of the calves available at 0 and 24 hours were analysed using a paired t-test (Table 2) i.e. the calves served as their own controls for treatment effect of OES. For assessment of OES effect on acid base status on normal calves, four clinically normal male calves (4 - 22 days) were obtained from the teaching hospital dairy unit and were force-fed $4 \mathrm{~L} \mathrm{OES}_{\text {exp }}$ after 12 hours fasting. Venous blood gases, blood electrolyte and total protein concentrations were analysed at $0,1,2,4,6,8,24$ hours post feeding. Using statprofile 9 technology we also tested 7 other commercial OES in duplicates for contents of electrolytes sodium, potassium, chloride and found similar discrepancies of measured values and published product values for the different electrolyte concentrations and the SID. The study was approved through review of an animal utilization protocol by University of Guelph animal care committee (\#434).

\section{Results}

The calves studied were on average 9 days old and had an average clinically estimated dehydration of $6.5 \%(5 \%-$ $8 \%$ ). All calves had severe diarrhea of 10 - 100 hours duration and were moderately depressed, for which treatment with oral electrolytes was indicated. Temperatures, heart rates, respiratory rates were generally at the upper level of normal values (Table 1). The average measured SID and electrolyte concentration of the OE$\mathrm{S}_{\exp }$ solutions tube-fed to the experimental calves were $[\mathrm{SID}]=88.3 \pm 4.7 \mathrm{mmol} / \mathrm{L}$, with $\left[\mathrm{Na}^{+}\right]=133 \pm 5.9$ $\mathrm{mmol} / \mathrm{L},\left[\mathrm{K}^{+}\right]=35 \pm 2.1 \mathrm{mmol} / \mathrm{Land}\left[\mathrm{Cl}^{-}\right]=81 \pm 4.0$ $\mathrm{mmol} / \mathrm{L}$. Propionate concentrations were not measured. The average in vitro $\mathrm{pH}$ of the solution fed was $6.6 \pm$ 0.15 . The metabolic acidosis observed in the plasma of these calves and reflected by measured $\mathrm{pH}$, SID, calculated $\mathrm{HCO}_{3}^{-}$, and base deficit was corrected significantly towards reference ranges $(\mathrm{p}<0.05)$ with two $2 \mathrm{~L}$ feedings in all but one calf (Table 2). Dehydration, as assessed quantitatively by alterations in total plasma protein (TP) and hematocrit, was also significantly corrected (Table 2). All calves were discharged from the clinics 24 to 72 hours post-admission. One calf in the discharged group was represented one week later with a complaint of ongoing diarrhea and recurrence of depression and dehydration. For the calves entered into the normal study Figure 2 shows plot for measured blood SID, refractometer total protein and calculated $\mathrm{HCO}_{3}$ concentrations. Calves showed mild abominal discomfort for 1 hour, with occasional kicking at their abdomen, shifting weight, and mildly elevated heart rate. From 1 to 4 hours all the calves continued to show periodic signs of discomfort and were mildly depressed. All the vital signs during this period were within normal ranges. No diarrhea and no neurologic signs were observed at any observation time. All venous parameters measured were within normal ranges at 24 hours after oral tubing (Figure 2). Figure 1 shows the expected and measured SID of the 8 OES using

Table 2. Within calf comparison of variables observed at admission and at 24 hours.

\begin{tabular}{|c|c|c|c|c|}
\hline Parameter & Admission $(n=10)$ & 24 hours $(n=10)$ & $\mathbf{p}^{\mathbf{a}}$ & Reference range $^{b}$ \\
\hline Base-deficit ${ }^{\mathrm{c}}(\mathrm{mmol} / \mathrm{L})$ & $-9.94 \pm 5.52$ & $0.24 \pm 5.98$ & 0.0014 & $0 \pm 2$ \\
\hline Bicarbonate $^{c}(\mathrm{mmol} / \mathrm{L})$ & $18.23 \pm 4.39$ & $27.16 \pm 5.27$ & 0.0007 & $20-28$ \\
\hline Lactate $(\mathrm{mmol} / \mathrm{L})$ & $2.67 \pm 2.49$ & $1.86 \pm 1.28$ & 0.3348 & $0.5-2.2$ \\
\hline $\mathrm{pH}$ & $7.17 \pm 0.07$ & $7.33 \pm 0.05$ & 0.0000 & $7.32-7.44$ \\
\hline $\mathrm{Na}^{+}(\mathrm{mmol} / \mathrm{L})$ & $130.4 \pm 5.08$ & $134.1 \pm 5.10$ & 0.0846 & $132-152$ \\
\hline $\mathrm{K}^{+}(\mathrm{mmol} / \mathrm{L})$ & $5.23 \pm 0.65$ & $5.34 \pm 1.11$ & 0.824 & $3.9-5.8$ \\
\hline $\mathrm{Cl}^{-}(\mathrm{mmol} / \mathrm{L})$ & $99.0 \pm 5.20$ & $98.3 \pm 5.24$ & 0.6102 & $99-110$ \\
\hline $\operatorname{SID}^{\mathrm{d}}(\mathrm{mmol} / \mathrm{L})$ & $35.04 \pm 3.84$ & $40.13 \pm 4.76$ & 0.0018 & $38-42$ \\
\hline Total $\operatorname{Protein}^{\mathrm{e}}(\mathrm{g} / \mathrm{L})$ & $70.9 \pm 11.24$ & $63.2 \pm 10.16$ & 0.0023 & $60-68$ \\
\hline $\operatorname{Hematocrit}^{\mathrm{f}}(\mathrm{L} / \mathrm{L})$ & $0.37 \pm 0.08$ & $0.33 \pm 0.07$ & 0.0000 & $0.22-0.38$ \\
\hline
\end{tabular}

${ }^{\mathrm{a}}$ Probability-value of paired t-test: $\mathrm{H}_{0}$ : Difference between means at admission and at 24 hours is zero; ${ }^{\mathrm{b}}$ Smith. Large Animal Internal Medicine 4 th edition 2009 Mosby-Elsevier; ${ }^{\mathrm{c}}$ Variable calculated by blood gas machine built in algorithm; ${ }^{\mathrm{d}} \mathrm{SID}$ measured $\mathrm{SID}=\left[\left(\mathrm{Na}^{+}+\mathrm{K}^{+}\right)-\left(\mathrm{Cl}^{-}+\mathrm{Lactate}^{-}\right)\right]$; ${ }^{\mathrm{e}} \mathrm{Variable}$ measured in plasma using refractometry; ${ }^{\mathrm{f}}$ Microhematocrit. 


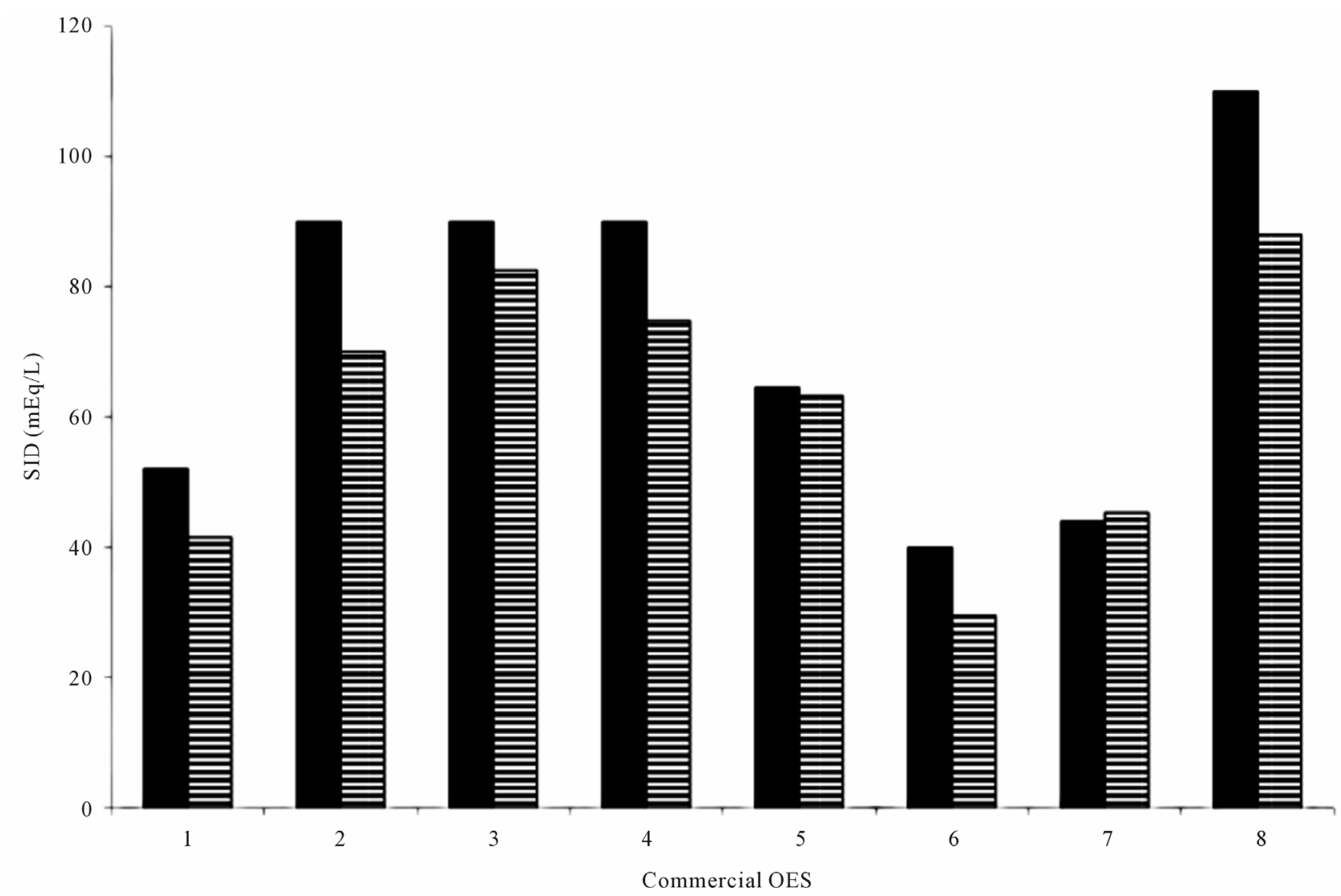

Figure 1. The strong ion difference of 7 commercial calf electrolyte solutions (1-7) and $\mathrm{OES}_{\mathrm{exp}}(8)$. SID $=(\mathrm{Na}+\mathrm{K})-(\mathrm{CI})$; with $\mathrm{Na}, \mathrm{K}$ and $\mathrm{Cl}$ measured with ion specific electrodes (stat profile 9 NovaBiomedical Canada); Horizontally striped bars: SID as measured with ion specific electrodes for $\mathrm{Na}, \mathrm{K}$ and $\mathrm{Cl}$. Solid black bars: SID concentration as in official product description.

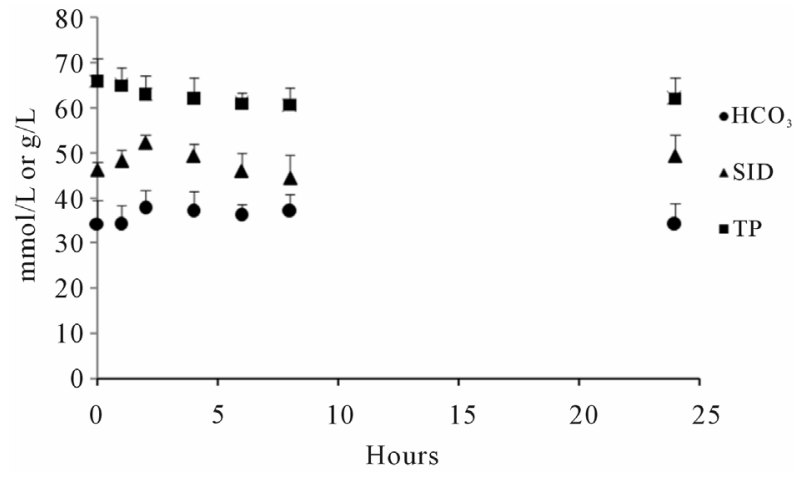

Figure 2. Mean SID Bicarbonate and total protein concentrations of 4 calves force-fed $4 \mathrm{~L} \mathrm{OES}_{\text {exp }} \triangle \mathrm{SID} \mathrm{mmol} / \mathrm{L}$; • Bicarbonate mmol/L; $\square$ Total protein (TP) g/L.

Statprofile 9 technology for concentration estimation.

\section{Discussion}

Traditionally, commercial OES must fulfil four requirements, including supply of adequate quantities of sodium to normalize ECF, contain agents that enhance absorption of sodium and water from intestines (e.g. short chain
VFA, glucose, glycine), provide an alkali agent, and ideally provide nutritional support [1]. This is supported by a recent study emphasizing that treatment of acidemia in sick calves, with or without diarrhea, should focus on intravenous or oral administration of a fluid containing sodium with a high effective SID [10]. The concentration of sodium in OES is empirical and in most available products is $\leq 120 \mathrm{mmol} / \mathrm{L}$. In published studies assessing OES treatments, the solutions containing $120 \mathrm{mmol} / \mathrm{L}$ of sodium outperformed those with lower sodium concentrations $[4,8]$. Interestingly the solutions containing 120 $\mathrm{mmol} / \mathrm{L}$ of sodium also contain the highest SID concentrations. The $\mathrm{OES}_{\text {exp }}$ tested in this study was theoretically formulated based on the suggested requirements for optimal OES performance in calves with naturally occurring diarrhea. The concentrations of electrolytes actually differed from the manufacturer's calculated concentrations used to prepare the $\mathrm{OES}_{\text {exp }}$ powder. Values for potassium and chloride were significantly higher than predicted by manufacturer. According to the manufacturer, it is very challenging to manufacture such oral electrolyte powder to the exact concentrations proposed. The manufacturer's "promised" SID concentrations of 
commercially available OES and $\mathrm{OES}_{\text {exp }}$ compared fairly well with the results of the statprofile 9 results. It should be emphasized that the statprofile 9 technology using ion specific electrodes is not a gold standard for true in vitro concentrations of electrolytes. The use of flame photometry would have been more accurate but was not available at the time of the study. The calves included in this study showed clinical and clinicopathologic criteria suitable for evaluating efficacy of the sodium propionate based $\mathrm{OES}_{\text {exp. }}$. The plasma sodium concentrations increased significantly with the two $2 \mathrm{~L} \mathrm{OES}_{\text {exp }}$ administrations. It appeared that two feedings were insufficient to completely correct the dehydration, and in some calves the acidosis. However, when compared to the group of our normal study calves, with a similar total OES load, treatment clearly had a greater effect on altering the SID and bicarbonate after 24 hours in those calves with diarrhea. This disparity was likely due to differences in renal water and electrolyte management in normovolemic calves in contrast to those with diarrhea that are hypovolemic and have disturbances in strong ions. Based on the partial yet incomplete response we observed, owners should be advised to continue OES feedings for at least $48-72$ hours or longer, depending on the clinical response and severity and persistence of the diarrhea [1]. In addition, owners should be advised to also feed milk or milk replacer alternatively to OES to calves with diarrhea. The majority of calves, which are not drinking and have diarrhea, will eventually develop body depletion of potassium, a very important electrolyte for cellular function and especially muscular function. Thus, most OES's, especially those that have been shown to be experimentally efficacious, contain at least $5-25 \mathrm{mmol} / \mathrm{L}$ of potassium. Theoretically, potassium will increase SID and thus, based on quantitative chemistry, the alkalinising potential of the solution $[1,6]$. Propionate, a readily and freely absorbed short chain fatty acid, was the "metabolizable base" in traditional acid-base interpretation, and has been shown to also facilitate sodium absorption in the small intestine. Moreover, it is usually readily and freely absorbed and funnelled in herbivores into the glucose metabolism [1,11]. Using quantitative acid base interpretation propionate is removed quickly from ECF, and metabolised as a volatile fatty acid to water and carbon dioxide. Therefore the net sodium load added to the system by the sodium propionate component is considered mechanistically to be the major driving force to replenish ECF and to correct strong ion acidosis by increasing SID $[10,12]$. The in vitro $\mathrm{pH}$ of the $\mathrm{OES}_{\exp }$ is 6.6 , which is equal to the $\mathrm{pH}$ of pure water at $37^{\circ} \mathrm{C}$. Only once propionate is absorbed by tissues will its effective in vivo SID of approximately $88 \mathrm{mEq} / \mathrm{L}$, attributable to the propionate accompanying sodium ion, kick in to correct acid base imbalances. That the strong ions are major players in acid base balance is also supported by work showing that isotonic or hypertonic $\mathrm{NaCl}$ can induce hyperchloremic acidosis in humans and animals [12]. Indeed, analysis of commercial electrolytes for SID concentrations reveals that increasing SID concentrations have greater alkalinising effect in healthy calves $[1,7]$. Thus, for the practicing veterinarian deciding which commercial OES to use or prescribe for young calves with diarrhea, it is helpful to also consider the sodium concentration and the SID of the product. Oral rehydration therapy in a calf with metabolic acidosis and dehydration should therefore start with a high SID OES. Then, once depression (a clinical indicator of acidosis) and dehydration have diminished, the OES should be switched to one with a low SID to avoid calves developing rebound alkalosis (personal observations). Fortunately, a normally functioning renal system usually readily compensates for sodium overload incurred with use of high SID oral electrolytes.

\section{Conclusion}

From this study it is concluded 1) that sodium propionate is a valid substitute for commonly used sodium base equivalents in oral electrolyte solutions and 2) that use of SID is a valid approach when formulating future OES. Just as it has been shown in recent years that oral dietary anion cation balance in dairy rations is important in managing calcium problems in dairy cows [13] the present study suggests that cation-anion balance is also important in OES for treatment of dehydration and strong ion acidosis in neonates.

\section{Acknowledgements}

Expert technical assistance was provided by Suzanne Misiaszek DVM, Bonnie Lambert and Neville Sookra DVM.

\section{REFERENCES}

[1] G. W. Smith, "Treatment of Calf Diarrhea: Oral Fluid Therapy," Veterinary Clinics of North America: Food Animal Practice, Vol. 25, No. 1, 2009, pp. 55-72. doi:10.1016/j.cvfa.2008.10.006

[2] P. D. Constable, "Fluid and Electrolyte Therapy in Ruminants," Veterinary Clinics of North America: Food Animal Practice, Vol. 19, No. 3, 2003, pp. 557-597.

[3] A. R. Michell, "Oral Rehydration for Diarrhoea: Symptomatic Treatment or Fundamental Therapy," Journal of Comparative Pathology, Vol. 118, No. 3, 1998, pp. 175193. doi:10.1016/S0021-9975(05)80125-2

[4] A. R. Michell, H. W. Brooks, D. G. White and A. J. Wagstaff, "The Comparative Effectiveness of Three Commercial Oral Solutions in Correcting Fluid, Electrolyte and Acid-Base Disturbances Caused by Calf Diar- 
rhea," British Veterinary Journal, Vol. 148, No. 6, 1992 , pp. 507-522.

[5] I. Lorenz, "D-Lactic Acidosis in Calves," The Veterinary Journal, Vol. 179, No. 2, 2009, pp. 197-203. doi:10.1016/j.tvjl.2007.08.028

[6] P. A. Stewart, "How to Understand Acid-Base: A Quantitative Acid-Base Primer for Biology and Medicine," Elsevier, New York, 1981

[7] P. D. Constable, P. G. Walker, D. E. Morin and J. H. Foreman, "Clinical and Laboratory Assessment of Hydration Status of Neonatal Calves with Diarrhea," Journal of the American Veterinary Medical Association, Vol. 212, No. 7, 1998, pp. 991-996.

[8] J. M. Naylor, L. Petrie, M. I. Rodriguez and P. Skilnick, "A Comparison of Three Oral Electrolyte Solutions in the Treatment of Diarrheic Calves," The Canadian Veterinary Journal, Vol. 31, No. 11, 1990, pp. 753-760.

[9] G. E. Rumbaugh, A. A. Ardans, D. Ginno and A. Trommershausen-Smith, "Measurement of Neonatal Equine Immunoglobulins for Assessment of Colostral Immunoglobulin Transfer: Comparison of Single Radial Immunodiffusion with the Zinc Sulfate Turbidity Test, Se- rum Electrophoresis, Refractometry for Total Serum Protein, and the Sodium Sulfite Precipitation Test," Journal of the American Veterinary Medical Association, Vol. 172, No. 3, 1978, pp. 321-325.

[10] P. D. Constable, H. R. Stämpfli, H. Navetat, et al., "Use of a Quantitative Strong Ion Approach to Determine the Mechanism for Acid-Base Abnormalities in Sick Calves with or without Diarrhea," Journal of Veterinary Internal Medicine, Vol. 19, No. 4, 2005, pp. 581-589.

[11] C. Demigné, C. Rémés, F. Chartier and D. Kaligis, "Utilization of Volatile Fatty Acids and Improvement of Fluid Therapy for Treatment of Dehydration in Diarrheic Calves," Annals of Veterinary Research, Vol. 14, No. 4, 1983, pp. 541-547.

[12] P. D. Constable, "Hyperchloremic Acidosis: the Classic Example of Strong Ion Acidosis," Anesthesia \& Analgesia, Vol. 96, No. 4, 2003, pp. 919-922.

[13] P. J. DeGaris, D. M. McNeil and E. Block, "Hypocalcemia in Dairy Cows: Meta-Analysis and Dietary Cation Anion Difference Theory Revisited," Journal of Dairy Science, Vol. 89, No. 2, 2006, pp. 669-684. doi: $10.3168 /$ jds.S0022-0302(06)72130-0 